\title{
Completion of radical hysterectomy does not improve survival of patients with cervical cancer and intraoperatively detected lymph node involvement: ABRAX international retrospective cohort study
}

\section{Cibula ${ }^{\text {a,* }}$, L. Dostalek ${ }^{\text {a }}$, P. Hillemanns ${ }^{\mathrm{b}}$, G. Scambia ${ }^{\mathrm{c}}$, J. Jarkovsky ${ }^{\mathrm{d}}$,} J. Persson ${ }^{\mathrm{e}}$, F. Raspagliesi ${ }^{\mathrm{f}}$, Z. Novak ${ }^{\mathrm{g}}$, A. Jaeger ${ }^{\mathrm{h}}$, M.E. Capilna ${ }^{\mathrm{i}}$, V. Weinberger ${ }^{j}$, J. Klat ${ }^{k}$, R.L. Schmidt ${ }^{1}$, A. Lopez ${ }^{\mathrm{m}}$, G. Scibilia ${ }^{\mathrm{n}}$, R. Pareja ${ }^{\circ}$, A. Kucukmetin ${ }^{\mathrm{p}}$, L. Kreitner ${ }^{\mathrm{q}}$, A. El-Balat ${ }^{\mathrm{r}}$, G.J.R. Pereira ${ }^{\mathrm{s}}$, S. Laufhütte ${ }^{\text {t }}$, D. Isla-Ortiz ${ }^{\text {u }}$, T. Toptas ${ }^{\text {v }, ~ B . ~ G i l-I b a n e z ~}{ }^{\text {w }}$, I. Vergote ${ }^{\text {x }}$, I. Runnenbaum ${ }^{\mathrm{y}}$

\footnotetext{
${ }^{a}$ Gynecologic Oncology Center, Department of Obstetrics and Gynecology, First Faculty of Medicine, Charles University and General University Hospital (Central and Eastern European Gynecologic Oncology Group, CEEGOG), Prague, Czech Republic

${ }^{\mathrm{b}}$ Department of Gynaecology and Obstetrics, Medical University Hannover, Hannover, Germany

${ }^{\mathrm{c}}$ Comprehensive Oncology Gynecology Operational Unit, Fondazione Policlinico Gemelli IRCCS (Italian Gynecological Oncology Group, MITO), Rome, Italy

${ }^{\mathrm{d}}$ Institute of Biostatistics and Analyses, Faculty of Medicine, Masaryk University, Brno, Czech Republic

${ }^{\mathrm{e}}$ Department of Obstetrics and Gynecology, Skaine University Hospital, Lund, Faculty of Medicine, Clinical Sciences, Lund University, Sweden

${ }^{\mathrm{f}}$ Fondazione IRCCS Instituto Nazionale Tumori (Italian Gynecological Oncology Group, MITO), Milan, Italy

${ }^{\mathrm{g}}$ Department of Gynecology, National Institute of Oncology (Central and Eastern European Gynecologic Oncology Group, CEEGOG), Budapest, Hungary

${ }^{\text {h }}$ University Medical Center Hamburg-Eppendorf (German Gynecological Oncology Group, AGO), Hamburg, Germany

${ }^{\mathrm{i}}$ First Obstetrics and Gynecology Clinic, University of Medicine and Pharmacy Targu Mures (Central and Eastern European Gynecologic Oncology Group, CEEGOG), Targu Mures, Romania

${ }^{\mathrm{j}}$ Department of Gynecology and Obstetrics, Faculty of Medicine, Masaryk University (Central and Eastern European Gynecologic Oncology Group, CEEGOG), Brno, Czech Republic

${ }^{\mathrm{k}}$ Department of Obstetrics and Gynecology, University Hospital Ostrava (Central and Eastern European Gynecologic Oncology Group, CEEGOG), Ostrava Poruba, Czech Republic

${ }^{1}$ Gynecologic Oncology Department, Barretos Cancer Hospital, Barretos, Brazil

${ }^{\mathrm{m}}$ Department of Gynecological Surgery, National Institute of Neoplastic Diseases, Lima, Peru

${ }^{\mathrm{n}}$ Cannizzaro Hospital (Italian Gynecological Oncology Group, MITO), Catania, Italy

${ }^{\circ}$ National Institute of Cancerology, Bogotá. Professor Universidad Pontificia Bolivariana, Medellín, Colombia

${ }^{\mathrm{p}}$ Northern Gynaecological Oncology Centre, Queen Elizabeth Hospital, Gateshead, United Kingdom

${ }^{\mathrm{q}}$ Gynecology Cancer Center, St. Franzis Hospital Munster (German Gynecological Oncology Group, AGO), Munster, Germany
}

* Corresponding author: Department of Obstetrics and Gynecology, Apolinarska 18, Prague 2, 12801, Czech Republic. E-mail address: dc@davidcibula.cz (D. Cibula). 
${ }^{\mathrm{r}}$ University Clinic Frankfurt, Goethe-University (German Gynecological Oncology Group, AGO), Frankfurt, Germany

${ }^{s}$ Gynecology Oncology Department, Institute of Cancerology - Las Americas, Clinic Medellin, Medellin, Colombia

${ }^{\mathrm{t}}$ Department of Obstetrics and Gynecology, District Hospital Altotting (German Gynecological Oncology Group, AGO), Altotting, Germany

u Gynecology Oncology Center, National Institute of Cancerology Mexico, Mexico

${ }^{v}$ Department of Gynecologic Oncology, Saglik Bilimleri University Antalya Research and Training Hospital, Antalya, Turkey

${ }^{\mathrm{w}}$ Unit of Gynaecological Oncology, Institute Clinic of Gynaecology, Obstetrics, and Neonatology (ICGON), Barcelona, Spain

${ }^{x}$ Department of Gynecology and Obstetrics, University Hospital Leuven, Leuven Cancer Institute (Belgium and Luxembourg Gynaecological Oncology Group, BGOG), Leuven, Belgium

${ }^{y}$ Department of Gynecology and Reproductive Medicine, Jena University Hospital, Friedrich Schiller University (German Gynecological Oncology Group, AGO), Jena, Germany

Received 15 September 2020; received in revised form 12 October 2020; accepted 28 October 2020

\section{KEYWORDS \\ Cervical cancer; Radical hysterectomy; Pelvic lymphadenectomy; Radical hysterectomy completion; Radical hysterectomy abandonment}

\begin{abstract}
Background: The management of cervical cancer patients with intraoperative detection of lymph node involvement remains controversial. Since all these patients are referred for (chemo)radiation after the surgery, the key decision is whether radical hysterectomy should be completed as originally planned, taking into account an additional morbidity associated with extensive surgical dissection prior to adjuvant treatment. The ABRAX study investigated whether completing a radical uterine procedure is associated with an improved oncological outcome of such patients.
\end{abstract}

Patients and methods: We performed retrospective analyses of 515 cervical cancer patients (51 institutions, 19 countries) who were referred for primary curative surgery between 2005 and 2015 (stage IA-IIB, common tumour types) in whom lymph node involvement was detected intraoperatively. Patients were stratified according to whether the planned uterine surgery was completed (COMPL group, $N=361$ ) or abandoned (ABAND group, $N=154$ ) to compare progression-free survival. Definitive chemoradiation was given to $92.9 \%$ patients in the ABAND group and adjuvant (chemo)radiation or chemotherapy to $91.4 \%$ of patients in the COMPL group.

Results: The risks of recurrence (hazard ratio [HR] 1.154, 95\% confidence intervals [CI] 0.799 $-1.666, P=0.45$ ), pelvic recurrence (HR $0.836,95 \%$ CI $0.458-1.523, P=0.56$ ), or death (HR $1.064,95 \%$ CI $0.690-1.641, P=0.78$ ) were not significantly different between the two groups. No subgroup showed a survival benefit from completing radical hysterectomy. Disease-free survival reached $74 \%$ (381/515), with a median follow-up of 58 months. Prognostic factors were balanced between the two groups. FIGO stage and number of pelvic lymph nodes involved were significant prognostic factors in the whole study cohort.

Conclusion: We showed that the completion of radical hysterectomy does not improve survival in patients with intraoperatively detected lymph node involvement, regardless of tumour size or histological type. If lymph node involvement is confirmed intraoperatively, abandoning uterine radical procedure should be considered, and the patient should be referred for definitive chemoradiation.

Clinical trials identifier: NCT04037124.

(C) 2020 Elsevier Ltd. All rights reserved.

\section{Introduction}

One of the most controversial steps in the treatment algorithm for the early stages of cervical cancer is the management of patients with intraoperatively detected positive pelvic lymph nodes. This situation used to be quite rare because preoperative modern imaging methods successfully identify the majority of patients with grossly enlarged lymph nodes and parametrial involvement, who are referred for definitive chemoradiation. Only in recent years has the intraoperative assessment of sentinel lymph node by frozen section become more common. This allows the detection of smaller-sized metastases, provides the surgeon with information about lymph node involvement at the beginning of the surgery and ultimately opens the 
possibility of modifying the management intraoperatively.

The 2018 guidelines for the management of patients with cervical cancer, developed by three European societies (European Society of Gynecological Oncology, European Society for Radiotherapy and Oncology and European Society of Pathology) recommend sentinel lymph node biopsy and frozen section pathological assessment as the first step in the management of all patients scheduled for primary curative surgery. If sentinel lymph node involvement is found intraoperatively, the guidelines recommend abandoning radical procedures and referring the patient for definitive chemoradiation [1]. However, this step in the guidelines is based on weak evidence from case-control and a few small cohort studies of only a maximum of a few dozen cases (Table S4). Therefore, the management of patients with intraoperatively detected lymph node involvement varies widely [1,2]. In a European Society of Gynaecological Oncology survey in 2018, $61 \%$ of respondents preferred to abandon radical hysterectomy [1]. By contrast, in a survey of Society of Gynecologic Oncology members, only $21 \%$ of respondents abandoned radical hysterectomy in patients with microscopic pelvic lymph node involvement, although the proportion of respondents increased for patients with gross pelvic involvement $(45 \%)$ or paraaortic lymph node involvement (69\%) [2].

If LN involvement is diagnosed intraoperatively, options of the management include completing or abandoning radical hysterectomy, performing or abandoning pelvic lymph node dissection and completing paraaortic lymph node dissection. The most important decision is whether to perform a radical uterine procedure (radical hysterectomy or radical trachelectomy), owing to the high morbidity associated with extensive surgical dissection in the pelvis followed by adjuvant pelvic radiotherapy [3]. In particular, a combination of radical surgery and radiotherapy increases the frequency and severity of surgical complications, such as urinary fistulas or lower-leg lymphedema [4-6], while surgery and radiotherapy are associated with different adverse event profiles $[7,8]$.

Data on the oncological outcomes of patients with lymph node involvement after radical hysterectomy and adjuvant radiotherapy are broadly available, with recent results showing five-year survival in stage IB at around $70 \%-85 \%[9,10]$. However, there are limited and inconsistent data for patients in whom radical hysterectomy was abandoned due to intraoperatively detected lymph node involvement. Prior studies included small, heterogeneous groups of patients, mostly with gross lymph node involvement that was not detected preoperatively due to the absence of modern imaging in the work-up [10-16]. Therefore, we established collaboration with international gynaecological centres that saw a high volume of cervical cancer to obtain retrospective data on a large cohort of patients with intraoperatively detected lymph node involvement who underwent either radical uterine procedure followed by adjuvant (chemo)radiotherapy or definitive chemoradiotherapy without a prior radical uterine procedure. We investigated whether the completion of radical uterine procedure improves recurrence-free and overall survival.

\section{Methods}

\subsection{Study design and participants}

The ABRAX (ABandoning RAdical hysterectomy in cerviX cancer) international, multicentre, retrospective cohort study studied the oncological outcomes of cervical cancer patients in whom radical hysterectomy was either completed as planned (COMPL) or abandoned (ABAND) following intraoperative detection of lymph node involvement. The ABRAX consortium was composed of 51 institutions across 19 countries in Europe, Central America and Latin America (Supplementary Table S2) and was led by the Central and Eastern European Gynecologic Oncology Group (CEEGOG).

Patients were retrospectively enrolled if they met the following inclusion criteria: (i) intraoperatively detected lymph node involvement; (ii) confirmed squamous cell carcinoma, adenocarcinoma or adenosquamous carcinoma; (iii) pretreatment disease stage IA-IIB [17]; (iv) primary surgery with a curative intent performed between January 2005 and December 2015 [18]. Lymph node involvement was defined as the presence of macrometastases (lesions $\geq 2 \mathrm{~mm}$ ) or micrometastases $(0.2-2 \mathrm{~mm})$ according to the TNM classification of malignant tumours $[17,19]$.

For intraoperative detection of lymph node involvement, patients were eligible regardless of the type of lymph node staging: gross lymph node involvement if confirmed by final pathology; microscopic involvement detected by frozen section (including sentinel lymph node biopsy only). Patients with any planned uterine procedures were eligible (including simple trachelectomy, radical trachelectomy, simple hysterectomy and radical hysterectomy), although most patients were scheduled for radical hysterectomy. Patients who underwent systematic pelvic lymphadenectomy and/or paraaortic lymphadenectomy were not excluded. Patients with preoperative evidence of lymph node involvement on imaging, other tumour types, negative final lymph node status, lymph node involvement reported by final pathology without intraoperative detection, and follow-up for less than 1 year were excluded.

The protocol was approved by the Institutional Review Board of the lead institution (General University 
Hospital in Prague, Czech Republic) in 2017. Institutional Review Board approval at the participating sites was a prerequisite for participation. Due to the retrospective nature of the study, the need for informed consent was waived by the Institutional Review Board. The study was performed in accordance with the Declaration of Helsinki. The ABRAX study was registered on ClinicalTrial.gov (NCT04037124) as ENGOTCx3/CEEGOG/ABRAX.

\subsection{Procedures and outcomes}

The principal investigator at each institution identified eligible patients, anonymised the data and transferred the data using a web-based system to ensure consistent data collection, which ended in June 2019. Detailed treatment information and primary oncological characteristics were extracted from the patients' medical reports.

Eligible patients with intraoperatively detected lymph node involvement were divided into the COMPL group or ABAND group (Supplementary Figure S1). The decision to complete or abandon surgery was at the discretion of the attending surgeon and institutional guidelines. Data on complications were reported when, according to the investigator's opinion, they were related to the treatment of cervical cancer. Adverse events were classified according to the NCI Common Terminology Criteria for Adverse Events Version (CTCAE) 4.0. Only events of grade 2 or higher were reported.

The primary endpoint of the study was recurrencefree survival, which was calculated from the time of surgery to the diagnosis of disease recurrence. Secondary endpoints were as follows: overall survival, pelvic recurrence-free survival, associations between prognostic factors and survival (tumour size, histological type, number of involved lymph nodes, type of metastasis and presence of lymphovascular space invasion) and prevalence of grade 2 or worse treatmentrelated adverse events (CTCAE 4.0).

\subsection{Statistical analyses}

Time to event data were calculated with the Kaplan-Meier method and compared using the logrank test. The Cox proportional hazard model was used to evaluate the influence of patient and treatment characteristics on time to event endpoints. Model results are presented as hazard ratios (HR) with 95\% confidence intervals (CI). Propensity score analysis was performed to estimate the effect of a treatment by accounting for the covariates between ABAND and COMPL group (surgical approach, pelvic lymphadenectomy performed, paraaortic lymph node involvement). It was computed using $\mathrm{R}$ with MatchIt package. Multivariate models were computed using the forward stepwise algorithm with the type of management forced into the model. Receiver operating characteristic curve analysis was used to identify the optimal cut-off values for continuous variables prior to their inclusion in regression modelling. Standard descriptive statistics were used, with absolute and relative frequencies for categorical variables and medians supplemented by the 5 th-95th percentile range for continuous variables, and were compared using Fisher's exact test and the Mann-Whitney $\mathrm{U}$ test, respectively. We adopted $\alpha=0.05$ (two-sided) as the level of statistical significance. The analyses were done using SPSS 25.0.0.1 (IBM Corporation).

\section{Results}

We analysed the data from 515 cervical cancer patients in whom lymph node involvement was detected during surgery performed between January 2005 and December 2015. The surgery was completed as planned in 361 patients (COMPL group) and abandoned in 154 patients (ABAND group). The preferred management strategy differed between the institutions without obvious time trends or geographical variations (Supplementary Tables S1 and S2). The proportion of patients in whom the procedure was abandoned ranged from $0 \%$ to $100 \%$ among the study sites (Supplementary Figure S2).

The characteristics of the 515 patients overall and by group are summarised in Table 1 . Among the 515 patients, lymph node involvement was macroscopic in 292 $(57 \%)$ and microscopic (detected on frozen section) in 223 (43\%). Macrometastases and micrometastases were found in $379(74 \%)$ and $136(26 \%)$ patients, respectively. Pelvic lymphadenectomy was completed in $489(95 \%)$ and paraaortic lymphadenectomy in $320(62 \%)$ patients. The majority of patients had pretreatment stage IB1 (287 patients, $56 \%$ ) [17], a largest tumour size of $2-4 \mathrm{~cm}$ (207 patients, 60\%) and squamous histological type (391 patients, 76\%) (Table 1).

The prevailing type of uterine surgery in the COMPL group was radical hysterectomy in 335 patients $(93 \%)$. All patients in the ABAND group underwent primary (chemo)radiation, while the majority received chemoradiation $(93 \%)$. Adjuvant treatment was administered to 330 of 361 patients (91\%) in the COMPL group, including chemoradiation in $266(74 \%)$, combined radiotherapy in $47(13 \%)$ and chemotherapy only in 17 $(5 \%)$.

Despite the retrospective study design, the ABAND and COMPL groups were well balanced in terms of major prognostic risk factors, including tumour size, pretreatment stage, tumour type and the type of the largest lymph node metastasis (Table 1). However, there were significant differences in some characteristics. In particular, an open surgical approach was used more 
Table 1

Comparison of patients with cervical procedure abandoned (ABAND) or completed (COMPL).

\begin{tabular}{|c|c|c|c|c|c|}
\hline \multirow[t]{2}{*}{ Characteristic } & & \multicolumn{2}{|c|}{ Cervical Procedure } & \multirow{2}{*}{$\begin{array}{l}\text { All } \\
\text { Patients } \\
(N=515)^{\mathrm{a}}\end{array}$} & \multirow[b]{2}{*}{$P^{\dagger}$} \\
\hline & & $\begin{array}{l}\operatorname{COMPL}(N= \\
361)^{\mathrm{a}}\end{array}$ & $\begin{array}{l}\text { ABAND }(N= \\
154)^{\mathrm{a}}\end{array}$ & & \\
\hline Age & & $48(29-69)$ & $46(29-70)$ & $47(29-70)$ & 0.76 \\
\hline BMI & $\mathrm{kg} / \mathrm{m}^{2}$ & $24(19-32)$ & $25(19-34)$ & $24(19-33)$ & 0.025 \\
\hline \multirow[t]{3}{*}{ ECOG performance status } & 0 & $258(83.5 \%)$ & $124(81.6 \%)$ & $\begin{array}{l}382 \\
(82.9 \%)\end{array}$ & 0.42 \\
\hline & 1 & $50(16.2 \%)$ & $26(17.1 \%)$ & $76(16.5 \%)$ & \\
\hline & 2 & $1(0.3 \%)$ & $2(1.3 \%)$ & $3(0.7 \%)$ & \\
\hline \multirow[t]{6}{*}{ Pretreatment stage (FIGO 2009) } & IA & $12(3.3 \%)$ & $3(1.9 \%)$ & $15(2.9 \%)$ & 0.17 \\
\hline & IB1 & $191(52.9 \%)$ & $96(62.3 \%)$ & $\begin{array}{l}287 \\
(55.7 \%)\end{array}$ & \\
\hline & IB2 & $57(15.8 \%)$ & $22(14.3 \%)$ & $79(15.3 \%)$ & \\
\hline & IIA & $39(10.8 \%)$ & $8(5.2 \%)$ & $47(9.1 \%)$ & \\
\hline & IIB & $41(11.4 \%)$ & $20(13.0 \%)$ & $61(11.8 \%)$ & \\
\hline & Unknown & $21(5.8 \%)$ & $5(3.2 \%)$ & $26(5.0 \%)$ & \\
\hline \multirow[t]{3}{*}{ Largest tumour size on radiological staging } & $<2 \mathrm{~cm}$ & $28(11.3 \%)$ & $16(16.0 \%)$ & $44(12.6 \%)$ & 0.08 \\
\hline & $2-4 \mathrm{~cm}$ & $143(57.7 \%)$ & $64(64.0 \%)$ & $\begin{array}{l}207 \\
(59.5 \%)\end{array}$ & \\
\hline & $>4$ & $77(31.0 \%)$ & $20(20.0 \%)$ & $97(27.9 \%)$ & \\
\hline \multirow[t]{4}{*}{ Tumour type } & Squamous & $269(74.6 \%)$ & $122(79.2 \%)$ & $\begin{array}{l}391 \\
(75.9 \%)\end{array}$ & 0.55 \\
\hline & Adenocarcinoma & $65(18.0 \%)$ & $22(14.3 \%)$ & $87(16.9 \%)$ & \\
\hline & Adenosquamous & $23(6.4 \%)$ & $10(6.5 \%)$ & $33(6.4 \%)$ & \\
\hline & Unknown & $4(1.1 \%)$ & $0(0.0 \%)$ & $4(0.8 \%)$ & \\
\hline \multirow[t]{2}{*}{$\begin{array}{l}\text { Macroscopic lymph node involvement found } \\
\text { intraoperatively }\end{array}$} & No & $150(41.6 \%)$ & $73(47.4 \%)$ & $\begin{array}{l}223 \\
(43.3 \%)\end{array}$ & 0.24 \\
\hline & Yes & $211(58.4 \%)$ & $81(52.6 \%)$ & $\begin{array}{l}292 \\
(56.7 \%)\end{array}$ & \\
\hline \multirow[t]{6}{*}{ Type of uterine procedure performed } & $\begin{array}{l}\text { Radical } \\
\text { hysterectomy }\end{array}$ & $335(92.8 \%)$ & $0(0.0 \%)$ & $\begin{array}{l}335 \\
(65.0 \%)\end{array}$ & $<0.0001$ \\
\hline & Simple hysterectomy & $14(3.9 \%)$ & $0(0.0 \%)$ & $15(2.9 \%)$ & \\
\hline & $\begin{array}{l}\text { Radical } \\
\text { trachelectomy }\end{array}$ & $9(2.5 \%)$ & $0(0.0 \%)$ & $9(1.7 \%)$ & \\
\hline & Simple trachelectomy & $2(0.6 \%)$ & $0(0.0 \%)$ & $2(0.4 \%)$ & \\
\hline & Unknown & $1(0.3 \%)$ & $0(0.0 \%)$ & $1(0.2 \%)$ & \\
\hline & Not performed & $0(0.0 \%)$ & $154(100.0 \%)$ & $\begin{array}{l}154 \\
(29.9 \%)\end{array}$ & \\
\hline \multirow[t]{5}{*}{ Surgical approach } & Open (laparotomy) & $251(69.5 \%)$ & $60(39.0 \%)$ & $\begin{array}{l}311 \\
(60.4 \%)\end{array}$ & $<0.0001$ \\
\hline & Robotic & $21(5.8 \%)$ & $20(13.0 \%)$ & $41(8.0 \%)$ & \\
\hline & Vaginal & $1(0.3 \%)$ & $0(0.0 \%)$ & $1(0.2 \%)$ & \\
\hline & Laparoscopic & $83(23 \%)$ & $67(43.5 \%)$ & $\begin{array}{l}150 \\
(29.1 \%)\end{array}$ & \\
\hline & Unknown & $5(1.4 \%)$ & $7(4.5 \%)$ & $12(2.3 \%)$ & \\
\hline \multirow[t]{3}{*}{ Pelvic lymphadenectomy performed } & No & $5(1.4 \%)$ & $18(11.7 \%)$ & $23(4.5 \%)$ & $<0.0001$ \\
\hline & Yes & $355(98.3 \%)$ & $134(87.0 \%)$ & $\begin{array}{l}489 \\
(95.0 \%)\end{array}$ & \\
\hline & Unknown & $1(0.3 \%)$ & $2(1.3 \%)$ & $3(0.6 \%)$ & \\
\hline \multirow[t]{3}{*}{ Paraaortic lymphadenectomy performed } & No & $150(41.6 \%)$ & $37(24.0 \%)$ & $\begin{array}{l}187 \\
(36.3 \%)\end{array}$ & $<0.0001$ \\
\hline & Yes & $206(57.1 \%)$ & $114(74.0 \%)$ & $\begin{array}{l}320 \\
(62.1 \%)\end{array}$ & \\
\hline & Unknown & $5(1.4 \%)$ & $3(1.9 \%)$ & $8(1.6 \%)$ & \\
\hline Paraaortic lymph node involvement & Yes & $30(17.0 \%)$ & $33(29.5 \%)$ & $63(21.9 \%)$ & 0.019 \\
\hline Recurrence & & $93(25.8 \%)$ & $41(26.6 \%)$ & $\begin{array}{l}134 \\
(26.0 \%)\end{array}$ & 0.74 \\
\hline Pelvic recurrence & & $45(12.5 \%)$ & $14(9.1 \%)$ & $59(11.5 \%)$ & 0.36 \\
\hline Death & & $71(19.7 \%)$ & $29(18.8 \%)$ & $\begin{array}{l}100 \\
(19.4 \%)\end{array}$ & 1.00 \\
\hline \multirow[t]{2}{*}{ Intraoperative complication } & No & $347(96.1 \%)$ & $149(96.8 \%)$ & $\begin{array}{l}496 \\
(96.3 \%)\end{array}$ & 1.00 \\
\hline & Yes & $14(3.9 \%)$ & $5(3.2 \%)$ & $19(3.7 \%)$ & \\
\hline
\end{tabular}


Table 1 (continued)

\begin{tabular}{|c|c|c|c|c|c|}
\hline \multirow{2}{*}{ Characteristic } & & \multicolumn{2}{|c|}{ Cervical Procedure } & \multirow{2}{*}{$\begin{array}{l}\text { All } \\
\text { Patients } \\
(N=515)^{\mathrm{a}}\end{array}$} & \multirow[b]{2}{*}{$P^{\dagger}$} \\
\hline & & $\begin{array}{l}\text { COMPL }(N= \\
361)^{\mathrm{a}}\end{array}$ & $\begin{array}{l}\text { ABAND }(N= \\
154)^{\mathrm{a}}\end{array}$ & & \\
\hline \multirow[t]{3}{*}{ Adverse events grade $\geq 2$ (before postoperative day 30 ) } & No & $332(92.0 \%)$ & $150(97.4 \%)$ & $\begin{array}{l}482 \\
(93.6 \%)\end{array}$ & 0.040 \\
\hline & Grade 2 & $13(3.6 \%)$ & $3(1.9 \%)$ & $16(3.1 \%)$ & \\
\hline & Grade 3 & $16(4.4 \%)$ & $1(0.6 \%)$ & $17(3.3 \%)$ & \\
\hline \multirow[t]{5}{*}{ Adverse events grade $\geq 2$ (since postoperative day 31 ) } & No & $330(91.4 \%)$ & $130(84.4 \%)$ & $\begin{array}{l}460 \\
(89.3 \%)\end{array}$ & $\mathbf{0 . 0 3 0}$ \\
\hline & Grade 2 & $17(4.7 \%)$ & $14(9.1 \%)$ & $31(6.0 \%)$ & \\
\hline & Grade 3 & $8(2.2 \%)$ & $8(5.2 \%)$ & $16(3.1 \%)$ & \\
\hline & Grade 4 & $6(1.7 \%)$ & $1(0.6 \%)$ & $7(1.4 \%)$ & \\
\hline & Grade 5 & $0(0.0 \%)$ & $1(0.6 \%)$ & $1(0.2 \%)$ & \\
\hline
\end{tabular}

Level of significance: $P<0.05$.

${ }^{\text {a }}$ Data are expressed as the median with 5th-95th percentile for continuous variables and absolute and relative frequencies for categorical variables. ${ }^{\dagger}$ Mann-Whitney $U$ test for continuous variables and Fisher exact test for categorical variables. COMPL $=$ cervical procedure completed as planned; ABAND = cervical procedure was abandoned; BMI = body mass index; ECOG = Eastern Cooperative Oncology Group.

often $(70 \%$ vs. $39 \% ; P<0.0001)$, pelvic lymphadenectomy was completed more frequently ( $98 \%$ vs. $87 \%$; $P<0.0001$ ), higher number of pelvic lymph node was removed (27 vs. $15 ; P<0.0001$ ), paraaortic lymphadenectomy was performed less frequently ( $57 \%$ vs. $74 \%$; $P<0.0001)$ and the number of patients with paraaortic pelvic lymph node involvement was lower $(17 \%$ vs. $30 \%$; $P=0.019)$ in the COMPL group.

Because some prognostic factors could only be assessed using specimens obtained after completing the cervical procedure, they were only determined in the COMPL group. In particular, 247 (68\%) had lymphovascular space invasion; parametrial invasion was found in $86(24 \%)$; and the vaginal margins were positive in 70 of 361 patients $(19 \%)$.

The patients in ABAND and COMPL groups were also matched 1-to-1 using propensity score matching to minimise the effect of clinically relevant variables with statistically significant difference between the groups. The analysis revealed that none of the outcome endpoints (recurrence, pelvic recurrence, survival) was influenced by the type of the management (completion/ abandonment of the cervical procedure) after removal of other potentially relevant covariates (Supplementary Figure S3).

Overall, with a median follow-up of 58 months, 134 patients experienced disease recurrence ( $26 \%$ of 515$)$, of which 59 experienced pelvic recurrence $(12 \%)$ and 100 patients died (19\% of 515) (Table 1). The Kaplan-Meier curves were not significantly different between the ABAND and COMP groups for recurrence-free survival $(P=0.45)$, pelvic recurrence-free survival $(P=0.56)$ or overall survival $(P=0.72)$ (Figure 1a-c). Furthermore, there were no significant differences between the ABAND and COMPL groups in terms of the risk of recurrence (HR 1.154, 95\% CI 0.799-1.666, $P=0.45$ ), pelvic recurrence (HR $0.836,95 \%$ CI $0.458-1.523$, $P=0.56$ ) or death (HR 1.064, 95\% CI 0.690-1.641,
$P=0.78)$ (Table 2). The type of management was not significantly associated with the risk of recurrence, pelvic recurrence or death in subgroup analyses, irrespective of pretreatment stage, radiologic tumour size, tumour type, number of pelvic lymph nodes involved, paraaortic lymph node involvement, surgical approach or performance of pelvic lymphadenectomy (Table 3). The only exception was a marginally higher risk of recurrence in the ABAND group among patients with stage IIB tumours (HR 2.270, 95\% CI 1.055-4.884, $P=0.036$ ). Even when the management type (cervical procedure abandoned) was forced into the multivariate models, it was not significantly associated with recurrence, pelvic recurrence or death (Table 4).

In the whole cohort, the risk of recurrence and pelvic recurrence was increased in patients with a tumour size of at least $4 \mathrm{~cm}$, patients with higher pretreatment stage and patients with more than one pelvic lymph node involved (Table 2). The presence of lymphovascular space invasion and the removal of at least 30 pelvic lymph nodes were marginally but significantly associated with the recurrence rate. Three factors were significantly associated with overall survival: tumour size of at least $4 \mathrm{~cm}$, higher pretreatment FIGO stage and removal of $\geq 30$ pelvic lymph nodes (Table 2 ). In the stepwise multivariate model, only pretreatment stage and number of pelvic lymph nodes involved were significantly associated with the risks of recurrence and pelvic recurrence, and only pretreatment stage was associated with survival (Table 4). The type of primary/ adjuvant treatment (chemotherapy, radiochemotherapy or combined radiotherapy) was not significant for the risk of recurrence in the multivariate model. In both ABAND and COMPL groups, majority of the patients underwent chemoradiation and in both groups recurrence risk reached 25\% (Supplementary Table S3). Survival was not significantly different between patients who underwent open or minimally 
invasive surgery $(P=0.590)$ (Supplementary Figure S4).
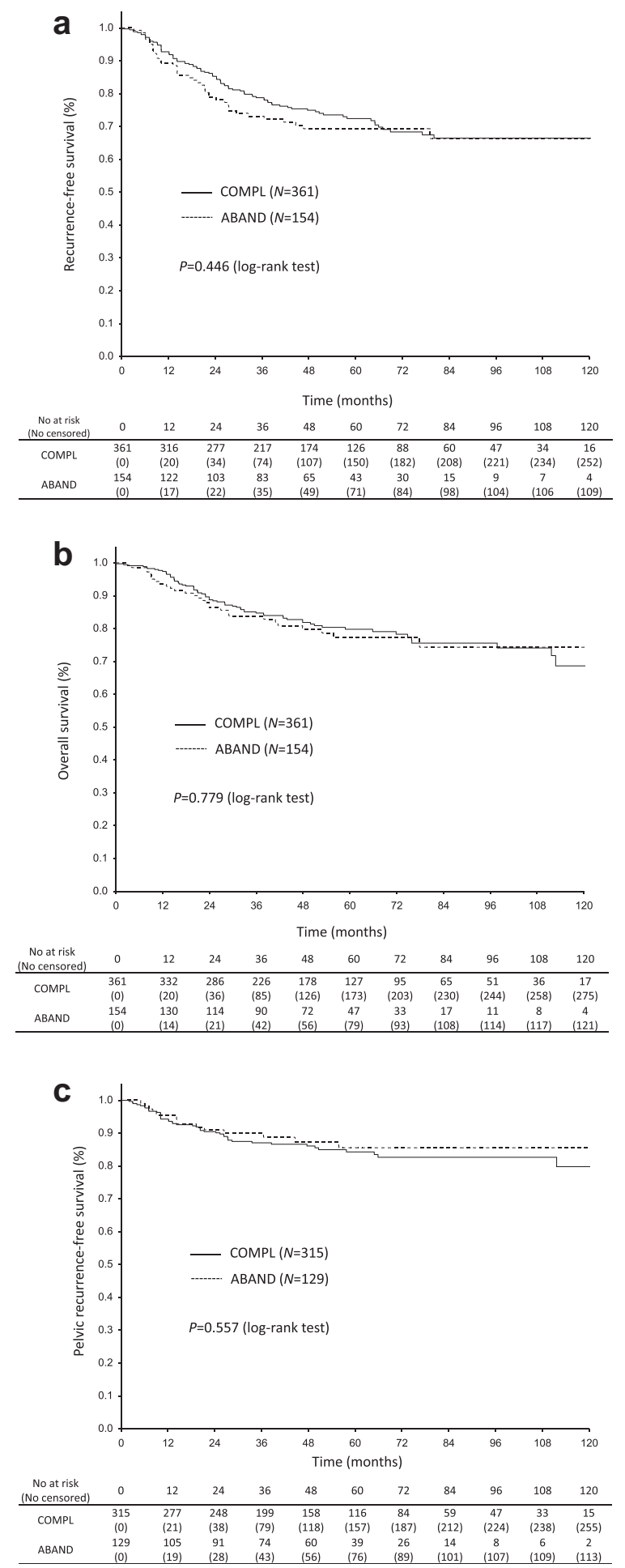

Fig. 1. Oncological outcomes according to the management strategy. (a) Recurrence-free survival. (b) Overall survival. (c) Pelvic recurrence-free survival. Time 0 was defined as the date of the scheduled operation in each patient. COMPL = cervical procedure completed as planned; ABAND = cervical procedure was abandoned.
The operation time was significantly longer (240 min vs. $172 \mathrm{~min} ; P<0.0001)$ and blood loss significantly greater $(350 \mathrm{ml}$ vs. $115 \mathrm{ml} ; P<0.0001)$ in the COMPL group than in the ABAND group. Postoperative complications of grade 2 or above within postoperative day 30 were more frequent in the COMPL group than in the ABAND group ( $8 \%$ vs. $3 \% ; P=0.040$ ). However, the frequency of treatment-related adverse events after postoperative day 30 were reported more frequently in the ABAND group than in the COMPL group $(16 \%$ vs. $9 \% ; P=0.030$ ).

\section{Discussion}

Our study represents the largest retrospective cohort of cervical cancer patients $(N=515)$ treated by primary curative surgery with intraoperatively detected lymph node involvement. When comparing two types of management, with and without completion of a radical uterine procedure, there was no difference in recurrence-free survival or overall survival in the whole study cohort or in subgroup analyses when patients were divided by major prognostic factors, including tumour size and tumour type. Several established risk factors were significantly associated with the survival outcomes in the whole cohort, including tumour size, FIGO stage and a higher number of positive pelvic lymph nodes. Disease-free survival of the whole cohort reached $74 \%$, which is consistent with the high-risk status of these patients with lymph node involvement.

Current clinical practice is heterogeneous in the management of patients with intraoperatively detected lymph node involvement. European guidelines recommend avoiding further pelvic surgery if lymph node involvement is detected intraoperatively [1]. However, this preference is not supported by strong evidence, because only case studies and small cohorts of patients in whom radical hysterectomy was abandoned have been reported to date (Supplementary Table S4). Completion of the cervical procedure was the prevailing management strategy in our study ( $70 \%$ of patients). We found no clear trend towards preferring either management strategy in any country nor region nor time trend over the period of patients' management (Supplementary Tables S1 and S2). This heterogeneous approach likely reflects the differing opinions of individual surgeons about the role of tumour removal before radiotherapy and the weak evidence supporting either management approach. Proponents of completing radical hysterectomy highlight a lower risk of central pelvis recurrence and a lower morbidity if brachytherapy is avoided. By contrast, proponents for abandoning radical surgery believe it reduces morbidity associated with the combination of radical surgery and adjuvant radiotherapy [18]. 
Table 2

Factors associated with the oncological outcomes in the whole cohort $(N=515)$.

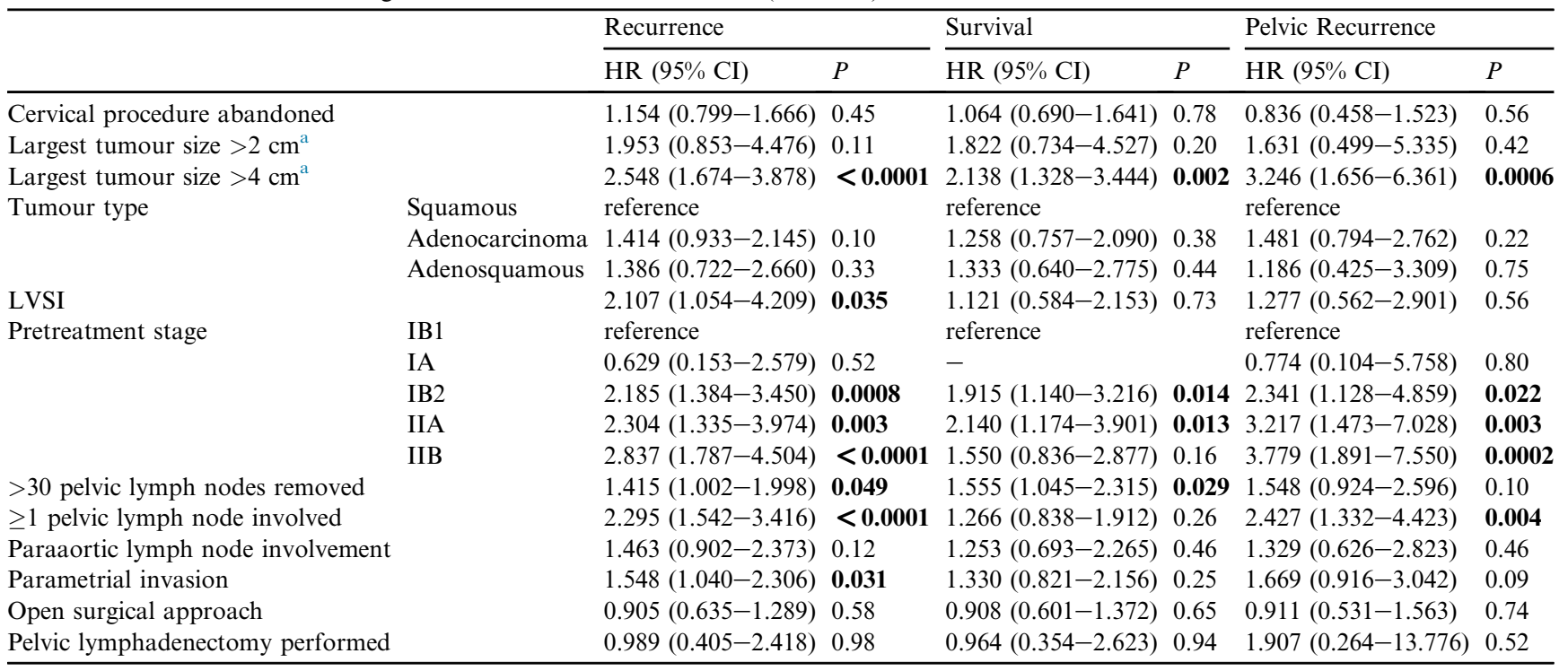

Level of significance: $P<0.05$.

${ }^{\text {a }}$ Radiological stage. HR $=$ hazard ratio; $\mathrm{CI}=$ confidence interval; LVSI = lymphovascular space invasion.

Several authors have attempted to address the role of completing radical hysterectomy in patients with lymph node involvement in the last 30 years, although the studies are subject to a variety of limitations and inconsistent conclusions (Supplementary Table S4). The outcomes of patients whose radical hysterectomy was abandoned were comparable with those in whom the procedure was completed in the studies by Potter et al. [11] and Richard et al. [10]. Trends towards lower recurrence-free survival following abandoned surgery were observed in other studies, although the differences did not reach statistical significance, probably due to the low number of patients [12,13]. Nevertheless, those studies were biased by unequal comparisons of high-risk patients with gross lymph node enlargement who are undoubtedly at a greater risk of treatment failure than patients with microscopic lymph node involvement detected on final pathology $[12,13]$ or patients without lymph node involvement [16].

Only one study compared the outcomes of patients in whom the decision to complete or abandon radical hysterectomy was made during the surgery [15]. That study evaluated the outcomes of 41 patients with gross lymph node involvement, in whom the procedure was abandoned in 15 patients and completed in 26 patients [15]. All of the patients were referred for chemoradiation after surgery. Although there were no significant differences between the groups, the local recurrence rate, distant recurrence rate and recurrence-free survival tended to be worse if radical hysterectomy was abandoned. More grade 3-4 complications were reported in the abandoned group ( $47 \%$ vs. $27 \%$ of cases), which the authors hypothesised to be due to increased radiation exposure [15]. In the most recent study, the authors compared the outcomes of 121 patients who underwent primary surgery in a 10 -year period at two sites in Amsterdam [20]. Patients with intraoperatively detected lymph node involvement, in whom radical hysterectomy was aborted, were compared with patients whose procedure was completed and lymph node involvement detected by final pathology. Although 5-year diseasefree survival was lower in patients with abandoned surgery, this difference was not significant after correcting for the number of positive lymph nodes in both groups. Patients in whom radical hysterectomy was abandoned represented a higher-risk group with a significantly greater number of lymph nodes involved, bilateral lymph node involvement and common iliac or paraaortic lymph node involvement. The frequency of grade 3 or 4 toxicities rate was also higher in the abandoned group ( $59 \%$ vs. $30 \%$ ). However, this difference was explained by a higher number of haematological toxicities because concomitant chemotherapy was administered more frequently in that group $(100 \%$ vs. $78 \%$ ). The authors concluded that better pelvic control could be achieved if radical hysterectomy is completed, although they admitted that a betterpowered study is necessary to compare the oncological and quality-of-life outcomes of the preferred management approach [20].

In the ABRAX study, we tried to avoid the limitations of previous studies, mainly by clearly defining the study cohort. In order to limit heterogeneity, we excluded patients whose cervical procedure was abandoned for a reason other than lymph node involvement. Furthermore, we only included patients if the decision to complete or abandon the cervical procedure was made during surgery. 
Table 3

Subgroup analysis of oncological outcomes according to the type of the management strategy.

\begin{tabular}{|c|c|c|c|c|}
\hline Category & & $N$ & HR $(95 \% \mathrm{CI})$ for abandoning the cervical procedure & $P$ \\
\hline \multicolumn{5}{|l|}{ Recurrence } \\
\hline \multirow[t]{2}{*}{ Largest tumour size $>2 \mathrm{~cm}$} & No & 44 & $1.861(0.375-9.235)$ & 0.45 \\
\hline & Yes & 304 & $1.119(0.691-1.812)$ & 0.65 \\
\hline \multirow[t]{2}{*}{ Largest tumour size $>4 \mathrm{~cm}$} & No & 251 & $1.310(0.725-2.367)$ & 0.37 \\
\hline & Yes & 97 & $1.218(0.579-2.562)$ & 0.60 \\
\hline \multirow[t]{3}{*}{ Tumour type } & Squamous & 389 & $1.287(0.841-1.970)$ & 0.25 \\
\hline & Adenocarcinoma & 87 & $0.679(0.276-1.671)$ & 0.40 \\
\hline & Adenosquamous & 33 & $1.436(0.371-5.566)$ & 0.60 \\
\hline \multirow[t]{5}{*}{ Pretreatment stage } & IB1 & 287 & $1.165(0.661-2.052)$ & 0.60 \\
\hline & IA & 15 & $4.899(0.302-79.447)$ & 0.26 \\
\hline & IB2 & 79 & $1.026(0.436-2.417)$ & 0.95 \\
\hline & IIA & 47 & $0.489(0.111-2.155)$ & 0.34 \\
\hline & IIB & 61 & $2.270(1.055-4.884)$ & 0.036 \\
\hline \multirow[t]{2}{*}{$>30$ pelvic lymph nodes removed } & No & 345 & $1.453(0.934-2.260)$ & 0.10 \\
\hline & Yes & 170 & $0.877(0.375-2.049)$ & 0.76 \\
\hline \multirow[t]{2}{*}{ Pelvic lymph node involvement } & No & 200 & $0.884(0.409-1.911)$ & 0.75 \\
\hline & Yes & 315 & $1.354(0.891-2.059)$ & 0.16 \\
\hline \multirow[t]{2}{*}{ Parametrial invasion } & No & 422 & $1.349(0.902-2.017)$ & 0.15 \\
\hline & Yes & 86 & - & \\
\hline \multirow{2}{*}{ Surgical approach: open } & No & 192 & $1.013(0.577-1.780)$ & 0.96 \\
\hline & Yes & 311 & $1.368(0.811-2.307)$ & 0.24 \\
\hline \multirow[t]{2}{*}{ Pelvic lymphadenectomy performed } & No & 23 & $0.912(0.102-8.175)$ & 0.94 \\
\hline & Yes & 489 & $1.160(0.788-1.706)$ & 0.45 \\
\hline \multirow[t]{2}{*}{ Paraaortic lymph node involvement } & No & 225 & $0.883(0.514-1.519)$ & 0.65 \\
\hline & Yes & 63 & $1.805(0.758-4.298)$ & 0.18 \\
\hline \multicolumn{5}{|l|}{ Survival } \\
\hline \multirow{2}{*}{ Largest tumour size $>2 \mathrm{~cm}$} & No & 44 & $1.119(0.187-6.702)$ & 0.90 \\
\hline & Yes & 304 & $1.020(0.586-1.775)$ & 0.94 \\
\hline \multirow[t]{2}{*}{ Largest tumour size $>4 \mathrm{~cm}$} & No & 251 & $1.093(0.566-2.111)$ & 0.79 \\
\hline & Yes & 97 & $1.170(0.472-2.903)$ & 0.73 \\
\hline Tumour type & Squamous & 389 & $1.262(0.773-2.061)$ & 0.35 \\
\hline & Adenocarcinoma & 87 & $0.490(0.142-1.696)$ & 0.26 \\
\hline & Adenosquamous & 33 & $0.920(0.185-4.565)$ & 0.92 \\
\hline Pretreatment stage & IB1 & 287 & $1.264(0.686-2.329)$ & 0.45 \\
\hline & IA & 15 & - & \\
\hline & IB2 & 79 & $1.306(0.501-3.403)$ & 0.59 \\
\hline & IIA & 47 & $0.258(0.033-2.017)$ & 0.20 \\
\hline & IIB & 61 & $1.567(0.508-4.832)$ & 0.43 \\
\hline$>30$ pelvic lymph nodes removed & No & 345 & $1.252(0.735-2.133)$ & 0.41 \\
\hline & Yes & 170 & $1.142(0.481-2.711)$ & 0.76 \\
\hline Pelvic lymph node involvement & No & 200 & $0.890(0.427-1.855)$ & 0.76 \\
\hline & Yes & 315 & $1.208(0.705-2.071)$ & 0.49 \\
\hline Parametrial invasion & No & 422 & $1.197(0.752-1.905)$ & 0.45 \\
\hline & Yes & 86 & - & \\
\hline Surgical approach: open & No & 192 & $1.359(0.705-2.619)$ & 0.36 \\
\hline & Yes & 311 & $0.870(0.442-1.710)$ & 0.69 \\
\hline Pelvic lymphadenectomy performed & No & 23 & $0.242(0.034-1.729)$ & 0.16 \\
\hline & Yes & 489 & $1.180(0.754-1.846)$ & 0.47 \\
\hline Paraaortic lymph node involvement & No & 225 & $0.705(0.365-1.363)$ & 0.30 \\
\hline & Yes & 63 & $1.328(0.470-3.753)$ & 0.59 \\
\hline Pelvic recurrence & & & & \\
\hline Largest tumour size $>2 \mathrm{~cm}$ & No & 44 & $0.920(0.083-10.161)$ & 0.95 \\
\hline & Yes & 304 & $0.703(0.288-1.715)$ & 0.44 \\
\hline Largest tumour size $>4 \mathrm{~cm}$ & No & 251 & $0.762(0.248-2.337)$ & 0.63 \\
\hline & Yes & 97 & $0.858(0.246-2.993)$ & 0.81 \\
\hline Tumour type & Squamous & 389 & $0.939(0.471-1.870)$ & 0.86 \\
\hline & Adenocarcinoma & 87 & $0.449(0.099-2.039)$ & 0.30 \\
\hline & Adenosquamous & 33 & $1.059(0.110-10.196)$ & 0.96 \\
\hline Pretreatment stage & IB1 & 287 & $0.580(0.195-1.725)$ & 0.33 \\
\hline & IA & 15 & - & \\
\hline & IB2 & 79 & $1.057(0.280-3.995)$ & 0.94 \\
\hline & IIA & 47 & $0.035(0.000-42.877)$ & 0.36 \\
\hline & IIB & 61 & $2.787(0.927-8.380)$ & 0.07 \\
\hline$>30$ pelvic lymph nodes removed & No & 345 & $0.956(0.465-1.964)$ & 0.90 \\
\hline
\end{tabular}


Table 3 (continued)

\begin{tabular}{lllll}
\hline Category & & $N$ & HR $(95 \%$ CI) for abandoning the cervical procedure & $P$ \\
\hline \multirow{3}{*}{ Pelvic lymph node involvement } & Yes & 170 & $0.868(0.260-2.902)$ & 0.82 \\
& No & 200 & $0.398(0.089-1.783)$ & 0.23 \\
Parametrial invasion & Yes & 315 & $1.100(0.568-2.132)$ & 0.78 \\
& No & 422 & $0.952(0.506-1.791)$ & 0.88 \\
Surgical approach: open & Yes & 86 & - & 0.38 \\
& No & 192 & $0.667(0.269-1.653)$ & 0.96 \\
Pelvic lymphadenectomy performed & Yes & 311 & $0.975(0.405-2.345)$ & 0.63 \\
Po & Yes & 23 & - & $0.86(0.463-1.597)$ \\
& No & 225 & $0.672(0.286-1.581)$ & 0.36 \\
\hline
\end{tabular}

Level of significance: $P<0.05$.

$\mathrm{HR}=$ hazard ratio; $\mathrm{CI}=$ confidence interval.

Of note, we found no significant differences between the ABAND and COMPL groups in the risks of recurrence $(P=0.45)$, pelvic recurrence $(P=0.56)$ or death $(P=0.78)$. Both groups were well balanced in the main prognostic factors, such as tumour size, pretreatment stage, tumour type and the type of the largest lymph node metastasis. Although there were significant differences between the groups in some parameters, including the proportion of patients with competed pelvic lymph node dissection, the number of pelvic lymph nodes harvested or the number of patients with paraaortic lymph node involvement, it is important to emphasise that those differences were in favour of the
COMPL group. Results were also confirmed using propensity matching analysis which account for covariates which may determine preference of the management (Supplementary Figure S3). The outcome of our study indicates that completing radical hysterectomy is not associated with a survival benefit in patients with intraoperatively detected lymph node involvement. Disease-free survival reached $74 \%(381 / 515)$ in the whole study cohort with a median follow-up of 58 months.

An important advantage of our study is the size of the cohort, which also allowed us, for the first time, to perform subgroup analyses. It was hypothesised that completion of radical hysterectomy before radiotherapy

Table 4

Multivariate models for oncological outcomes with a stepwise algorithm and type of management forced into the model.

\begin{tabular}{|c|c|c|c|}
\hline & & HR $(95 \% \mathrm{CI})$ & $P$ \\
\hline \multicolumn{4}{|l|}{ Recurrence } \\
\hline Cervical procedure abandoned (forced) & & $1.309(0.895-1.915)$ & 0.17 \\
\hline \multirow[t]{6}{*}{ Pretreatment stage } & IB1 & Reference & \\
\hline & IA & $0.740(0.180-3.049)$ & 0.68 \\
\hline & IB2 & $2.064(1.296-3.285)$ & 0.002 \\
\hline & IIA & $2.309(1.314-4.056)$ & 0.004 \\
\hline & IIB & $2.408(1.500-3.865)$ & 0.0003 \\
\hline & Unknown & $1.994(0.851-4.676)$ & 0.11 \\
\hline$>1$ pelvic lymph node involved & & $2.267(1.491-3.446)$ & 0.0001 \\
\hline \multicolumn{4}{|l|}{ Survival } \\
\hline Cervical procedure abandoned (forced) & & $1.194(0.772-1.849)$ & 0.43 \\
\hline \multirow[t]{6}{*}{ Pretreatment stage } & IB1 & Reference & \\
\hline & IA & - & \\
\hline & IB2 & $1.851(1.092-3.139)$ & 0.022 \\
\hline & IIA & $2.252(1.232-4.114)$ & 0.008 \\
\hline & IIB & $1.529(0.824-2.838)$ & 0.18 \\
\hline & Unknown & $1.924(0.820-4.514)$ & 0.13 \\
\hline \multicolumn{4}{|l|}{ Pelvic recurrence } \\
\hline Cervical procedure abandoned (forced) & & $0.981(0.523-1.840)$ & 0.95 \\
\hline \multirow[t]{6}{*}{ Pretreatment stage } & IB1 & Reference & \\
\hline & IA & $0.892(0.119-6.682)$ & 0.91 \\
\hline & IB2 & $2.132(0.996-4.561)$ & 0.05 \\
\hline & IIA & $3.245(1.470-7.161)$ & 0.004 \\
\hline & IIB & $3.073(1.491-6.333)$ & 0.002 \\
\hline & Unknown & $3.252(1.099-9.619)$ & 0.033 \\
\hline$>1$ pelvic lymph node involved & & $2.340(1.235-4.434)$ & 0.009 \\
\hline
\end{tabular}

Level of significance: $P<0.05$.

$\mathrm{HR}=$ hazard ratio; $\mathrm{CI}=$ confidence interval. 
could be beneficial in larger tumours or in less radiosensitive adenocarcinomas [21]. However, our study did not find any significant benefit of completing radical surgery in any tumour type or size.

The main limitation of the study is its retrospective design, which might introduce a selection bias. However, prospective studies in this context are very unlikely, if not unrealistic. Considering the recurrence rate reported in this study $(26 \%)$, more than 3500 cases would need to be randomised in a prospective study with a power of $80 \%$ and a non-inferiority margin of $5 \%$. Considering the prevalence of lymph node involvement about $12 \%$, it would be necessary to screen more than 30,000 patients with early-stage cervical cancer. Therefore, the collection of a large cohort of patients in the ABRAX study, thanks to the collaboration of 50 gynaecological oncological centres across 19 countries, provides the best available evidence for assessing this task. Another limitation of our study is the difficulty to retrospectively evaluate postoperative morbidity, especially long-term adverse events after adjuvant therapy. The very low prevalence of long-term complications after postoperative day 30 in our study indicates these events are underreported. To our knowledge, no prospective study has compared the quality of life after radical surgery, definitive chemoradiation and combined treatment. Nevertheless, a combination of multiple procedures is certainly associated with higher morbidity. Adjuvant radiotherapy increases the risk and worsens the manifestation of certain surgical complications, such as urinary fistula and lower-leg lymphedema [4,5]. Furthermore, surgery and radiotherapy are associated with different types of complications. This has been confirmed by retrospective studies evaluating the quality of life in cervical cancer survivors, which consistently showed that patients who received radiotherapy have the highest risk of bladder and bowel dysfunction, sexual dysfunction, psychological consequences and severe impairment of quality of life [7,8,22,23]. When comparing late toxicity in stage IIB cervical cancer patients after radical surgery followed by radiotherapy versus definitive radiotherapy, combined treatment led to significantly increased grade $3-4$ late toxicity $(22.1 \%$ vs. $10.6 \%, P=0.048$ ) including small bowel obstruction or lymphedema [24]. Similarly, significantly increased grade $3-4$ acute gastrointestinal toxicity $(P=0.036)$ and grade $3-4$ lower limb lymphedema $(P=0.017)$ was observed after combined treatment versus primary radiotherapy group in FIGO IIB cervical cancer patients [25]. Therefore, although our study did not allow us to reliably assess post-treatment morbidity, completion of radical hysterectomy can hardly be advocated by the improvement of quality of life after combined treatment in comparison with primary chemoradiation.

Our study was not designed to address the role of systematic pelvic lymphadenectomy. However, it is unlikely that removing lymph nodes with a normal appearance on preoperative staging improves prognosis. Systematic lymphadenectomy is not routinely performed before definitive chemoradiation even in patients with lymph node involvement on imaging. Therefore, a striking finding of our study is that pelvic lymphadenectomy was completed in $87 \%$ of patients in the ABAND group. This likely reflects the common practice of surgeons removing the pelvic lymph nodes while waiting for the results of frozen sections.

\section{Conclusion}

In conclusion, completing radical hysterectomy did not improve the prognosis of patients with intraoperatively detected lymph node involvement. Furthermore, the risk of recurrence was not decreased irrespective of tumour size, histological type or other traditional risk factors. Therefore, if pelvic lymph node involvement is found during surgery, abandoning the planned uterine procedure should be considered, and the patient should be referred for definitive chemoradiation.

\section{Funding}

This study was supported by Charles University in Prague [UNCE 204065 to D.C., PROGRES Q28/LF1 to D.C.]; and the Czech Health Research Council [NV1903-0023 to D.C.].

\section{Role of the funding source}

The funding bodies were not involved in study design, data collection, data analysis, data interpretation or writing of the report.

\section{Author contributions}

DC and LD designed the study. All authors were involved in data acquisition. JJ performed the statistical analyses. DC and LD were involved in data analyses. DC and LD drafted the manuscript. All authors were involved in revising the work for intellectual content. All authors approved the final version of the paper for publication.

\section{Conflict of interest statement}

The authors declare that there are no conflicts of interest.

\section{Acknowledgements}

We would like to acknowledge the investigators from all 51 sites participating in the ABRAX study. (Bizzarri Nicolo, Northern Gynaecological Oncology Centre, Queen Elizabeth Hospital, Gateshead, United 
Kingdom; Bogani Giorgio, Fondazione IRCCS Istituto Nazionale Tumori - Milan, Milan, Italy; Bronger Holger, Clinic for Gynaecology, Right-hand side of the River Isar Hospital, University Hospital of the Technical University of Munich, Munich, Germany; Di Martino Giampaolo, Department of Obstetrics and Gynecology, Unit of Gynecologic Oncology Surgery, San Gerardo Hospital, Monza, Italy; Danese Saverio, Department of Gynecology and Obstetrics, Sant'Anna Hospital, Torino, Italy; De Amicis Andrea, Comprehensive Oncology Gynecology Operational Unit, Fondazione Policlinico Gemelli IRCCS, Rome, Italy; De Iaco Pierandrea, Gynecologic Onclogy Unit, S. Orsola Malpighi Hospital, Bologna, Italy; Denschlag Dominik, Hochtaunus-Clinic Bad Homburg; Women's Hospital, Bad Homburg, Germany; Deryal Mustafa, Gynaecological Cancer Center, Caritas Hospital Saarbrücken, Saarbrücken, Germany; Ekdahl Linnea, Department of Obstetrics and Gynecology, Skane University Hospital, Lund, Sweden; Fagotti Anna, Comprehensive Oncology Gynecology Operational Unit, Fondazione Policlinico Gemelli IRCCS, Rome, Italy; Felberbaum Ricardo, Clinic of Kempten, Clinic Association Allgäu, department of Obstetrics and Gynecology, Kempten, Germany; Fischerova Daniela, Gynecologic Oncology Center, Department of Obstetrics and Gynecology, First Faculty of Medicine, Charles University and General University Hospital, Prague, Czech Republic; Fruhauf Filip, Gynecologic Oncology Center, Department of Obstetrics and Gynecology, First Faculty of Medicine, Charles University and General University Hospital, Prague, Czech Republic; Gil-Ibanez Blanca, Unit of Gynecological Oncology, Institute Clinic of Gynaecology, Obstetrics, and Neonatology, Hospital ClinicInstitut d'Investigacions Biomediques August $\mathrm{Pi}$ Sunyer (IDIBAPS), University of Barcelona, Barcelona, Spain; Greggi Stefano, Department of Gynecology Oncology, National Institute for the Study and Treatment of Tumors in Naples, Naples, Italy; Hager Dietrich, Gynecology Cancer Center, Clinic for Gynaecology and Obstetrics, Thuringen-Clinic "Georgius Agricola" GmbH, Saalfeld, Germany; Halder Christina, St. Marien Clinic, Women's Hospital, Amberg, Germany; Hanker Lars, Clinic for Gynaecology and Obstetrics, University Hospital SchleswigHolstein, Lübeck, Germany; Herboltova Petra, Department of Obstetrics and Gynecology, Jihlava Hospital, Jihlava, Czech Republic; Kascak Peter, Department of Obstetrics and Gynecology, University Hospital Trencin, Trencin, Slovakia; Knapp Pawel, Department of Gynecology and Gynecologic Oncology, Medical Uniwersity of Bialystok, Bialystok, Poland; Kocian Roman, Gynecologic Oncology Center, Department of Obstetrics and Gynecology, First Faculty of Medicine, Charles University and General University Hospital, Prague, Czech Republic; Krkoska Milan, Department of Gynecological Oncology,
National Oncology Institute, Bratislava, Slovakia; Latos Kunibert, Clinic for Gynaecology and Obstetrics, Katharinen Hospital Unna, Unna, Germany; Muller Andreas, City Clinic Karlsruhe, Women's Hospital, Karlsruhe, Germany; Nestle-Kramling Carolin, Evangelical Hospital Dusseldorf, Women's Clinic, Dusseldorf, Germany; Novotny Zdenek, Department of Gynaecology and Obstetrics, University Hospital Pilsen, Charles University, Prague, Czech Republic; Presl Jiri, Department of Gynaecology and Obstetrics, University Hospital Pilsen, Charles University, Prague, Czech Republic; Roccio Marianna, Department of Obstetrics and Gynecology, Fondazione IRCCS Policlinico San Matteo, Pavia, Italy; Roztočil Aleš, Department of Obstetrics and Gynecology, Jihlava Hospital, Jihlava, Czech Republic; Schwenzer Thomas, Clinic Dortmund gGmbH-Clinic Centre Mitte, Women's Hospital, Dortmund, Germany; Sanchez Octavio Arencibia, Department of Gynecologic Oncology, University Hospital of the Canary Islands, Las Palmas de Gran Canaria, Spain; Stepanyan Artem, Gynecological Oncology Service, NAIRI Medical Center, Yerevan, Armenia; Tsepelidou Eleni, Evangelical Hospital Dusseldorf, Women's clinic, Dusseldorf, Germany; Ureyen Isin, Department of Gynecologic Oncology, Saglik Bilimleri University Antalya Research and Training Hospital, Antalya, Turkey; Sarah Ehmann, KEM, Essen, Germany; Slama Jiri, Gynecologic Oncology Center, Department of Obstetrics and Gynecology, First Faculty of Medicine, Charles University and General University Hospital, Prague, Czech Republic; Struk Svitlana, Gynecology Department, Lviv State Regional oncology treatment and diagnostic Center, Lviv, Ukraine; Stukan Maciej, Gdynia Oncology Center, Department of Gynecologic Oncology, Pomeranian Hospitals, Gdynia, Poland; Tsolko Oleh, Gynecology Department, Lviv State Regional oncology treatment and diagnostic Center, Lviv, Ukraine; Volodko Natalia, Gynecology Department, Lviv State Regional oncology treatment and diagnostic Center, Lviv, Ukraine; Zapardiel Ignacio, Gynecologic Oncology Unit, La Paz University Hospital, Madrid, Spain; Perrone Anna Myriam, Gynecologic Oncology Unit, S. Orsola - Malpighi Hospital, Bologna, Italy.)

\section{Appendix A. Supplementary data}

Supplementary data to this article can be found online at https://doi.org/10.1016/j.ejca.2020.10.037.

\section{References}

[1] Cibula D, Potter R, Planchamp F, Avall-Lundqvist E, Fischerova D, Haie Meder C, et al. The European society of gynaecological oncology/European society for radiotherapy and oncology/European society of pathology guidelines for the 
management of patients with cervical cancer. Radiother Oncol 2018;127:404-16.

[2] Garg G, Shah JP, Toy EP, Field JB, Bryant CS, Liu JR, et al. Intra-operative detection of nodal metastasis in early stage cervical cancer: a survey of the practice patterns of SGO members. Gynecol Oncol 2011;121:143-7.

[3] Le Borgne G, Mercier M, Woronoff AS, Guizard AV, Abeilard E, Caravati-Jouvenceaux A, et al. Quality of life in long-term cervical cancer survivors: a population-based study. Gynecol Oncol 2013;129:222-8.

[4] Bilek K, Ebeling K, Leitsmann H, Seidel G. Radical pelvic surgery versus radical surgery plus radiotherapy for stage Ib carcinoma of the cervix uteri. Preliminary results of a prospective randomized clinical study. Archiv fur Geschwulstforschung 1982; 52:223-9.

[5] Rotman M, Sedlis A, Piedmonte MR, Bundy B, Lentz SS, Muderspach LI, et al. A phase III randomized trial of postoperative pelvic irradiation in Stage IB cervical carcinoma with poor prognostic features: follow-up of a gynecologic oncology group study. Int J Radiat Oncol Biol Phys 2006;65:169-76.

[6] Ryan M, Stainton MC, Slaytor EK, Jaconelli C, Watts S, Mackenzie P. Aetiology and prevalence of lower limb lymphoedema following treatment for gynaecological cancer. Aust N Z J Obstet Gynaecol 2003;43:148-51.

[7] Bjelic-Radisic V, Jensen PT, Vlasic KK, Waldenstrom AC, Singer S, Chie W, et al. Quality of life characteristics inpatients with cervical cancer. Eur J Canc 2012;48:3009-18.

[8] Park SY, Bae DS, Nam JH, Park CT, Cho CH, Lee JM, et al. Quality of life and sexual problems in disease-free survivors of cervical cancer compared with the general population. Cancer 2007; 110:2716-25.

[9] Cibula D, Abu-Rustum NR, Dusek L, Zikan M, Zaal A, Sevcik L, et al. Prognostic significance of low volume sentinel lymph node disease in early-stage cervical cancer. Gynecol Oncol 2012;124:496-501.

[10] Richard SD, Krivak TC, Castleberry A, Beriwal S, Kelley 3rd JL, Edwards RP, et al. Survival for stage IB cervical cancer with positive lymph node involvement: a comparison of completed vs. abandoned radical hysterectomy. Gynecol Oncol 2008;109:43-8.

[11] Potter ME, Alvarez RD, Shingleton HM, Soong SJ, Hatch KD. Early invasive cervical cancer with pelvic lymph node involvement: to complete or not to complete radical hysterectomy? Gynecol Oncol 1990;37:78-81.

[12] Suprasert P, Srisomboon J, Charoenkwan K, Siriaungul S, Khunamornpong S, Siriaree S, et al. Outcomes of abandoned radical hysterectomy in patients with stages IB-IIA cervical cancer found to have positive nodes during the operation. Int J Gynecol Canc 2005; 15:498-502.

[13] Gray HJ, Seifert E, Sal YRVG, Nicandri KF, Koh WJ Goff BA. The abandoned radical hysterectomy for cervical cancer: clinical predictors and outcomes. Obstetr Gynecol Int 2010;2010:743794.

[14] Whitney CW, Stehman FB. The abandoned radical hysterectomy: a gynecologic oncology group study. Gynecol Oncol 2000;79: $350-6$.

[15] Ziebarth AJ, Smith H, Killian ME, Nguyen NA, Durst JK, Subramaniam A, et al. Completed versus aborted radical hysterectomy for node-positive stage IB cervical cancer in the modern era of chemoradiation therapy. Gynecol Oncol 2012;126: 69-72.

[16] Bremer GL, van der Putten HW, Dunselman GA, de Haan J. Early stage cervical cancer: aborted versus completed radical hysterectomy. Eur J Obstet Gynecol Reprod Biol 1992;47: $147-51$.

[17] Pecorelli S. Revised FIGO staging for carcinoma of the vulva, cervix, and endometrium. Int J Gynaecol Obstet 2009;105: $103-4$.

[18] Dostalek L, Runnebaum I, Raspagliesi F, Vergote I, Dusek L, Jarkovsky J, et al. Oncologic outcome after completing or abandoning (radical) hysterectomy in patients with cervical cancer and intraoperative detection of lymph node positivity; ABRAX (ABandoning RAd hyst in cerviX cancer). Int J Gynecol Canc 2020;30:261-4.

[19] Bhatla N, Berek JS, Cuello Fredes M, Denny LA, Grenman S, Karunaratne K, et al. Revised FIGO staging for carcinoma of the cervix uteri. Int J Gynaecol Obstet 2019;145:129-35.

[20] Derks M, Groenman FA, van Lonkhuijzen L, Schut PC, Westerveld H, van der Velden J, et al. Completing or abandoning radical hysterectomy in early-stage lymph node-positive cervical cancer: impact on disease-free survival and treatment-related toxicity. Int J Gynecol Canc 2017;27:1015-20.

[21] Landoni F, Maneo A, Colombo A, Placa F, Milani R, Perego P, et al. Randomised study of radical surgery versus radiotherapy for stage Ib-IIa cervical cancer. Lancet 1997:350:535-40.

[22] Derks M, van Lonkhuijzen LR, Bakker RM, Stiggelbout AM, de Kroon CD, Westerveld $\mathrm{H}$, et al. Long-term morbidity and quality of life in cervical cancer survivors: a multicenter comparison between surgery and radiotherapy as primary treatment. Int J Gynecol Canc 2017;27:350-6.

[23] Frumovitz M, Sun CC, Schover LR, Munsell MF, Jhingran A, Wharton JT, et al. Quality of life and sexual functioning in cervical cancer survivors. J Clin Oncol 2005;23:7428-36.

[24] Mabuchi S, Okazawa M, Isohashi F, Matsuo K, Ohta Y, Suzuki O, et al. Radical hysterectomy with adjuvant radiotherapy versus definitive radiotherapy alone for FIGO stage IIB cervical cancer. Gynecol Oncol 2011;123:241-7.

[25] Chai Y, Wang T, Wang J, Yang Y, Gao Y, Gao J, et al. Radical hysterectomy with adjuvant radiotherapy versus radical radiotherapy for FIGO stage IIB cervical cancer. BMC Canc 2014;14: 63. 